\title{
Topological phase structure of vector vortex beams
}

\author{
C. E. R. Souza ${ }^{1}$, J. A. O. Huguenin ${ }^{2}$, and A. Z. Khoury ${ }^{1}$ \\ 1 Instituto de Fúsica, Universidade Federal Fluminense, 24210-346, Niterói - RJ, Brazil and \\ 2 Instituto de Ciências Exatas, Universidade Federal Fluminense, 27213-415, Volta Redonda - RJ, Brazil
}

\begin{abstract}
The topological phase acquired by vector vortex optical beams is investigated. Under local unitary operations on their polarization and transverse degrees of freedom, the vector vortices can only acquire discrete geometric phase values, 0 or $\pi$, associated with closed paths belonging to different homotopy classes on the $\mathrm{SO}(3)$ manifold. These discrete values are demonstrated through interferometric measurements and the spin-orbit mode separability is associated to the visibility of the interference patterns. The local unitary operations performed on the vector vortices involved both polarization and transverse mode transformations with birefringent wave plates and astigmatic mode converters. The experimental results agree with our theoretical simulations and generalize our previous results obtained with polarization transformations only.
\end{abstract}

PACS numbers: PACS: 03.65.Vf, 03.67.Mn, 07.60.Ly, 42.50.Dv

\section{INTRODUCTION}

Since the seminal works by Pancharatnam [1, 2], Aharonov 3] and Berry [4], the concepts of geometric and topological phases found numerous applications in different contexts. Besides its intrinsic beauty, geometric phases are candidates for robust implementations of quantum computing gates [ $[\underline{5}, 6]$. The geometric phase acquired by optical vortices under cyclic transformations was theoretically predicted by van Enk 7] and interpreted in terms of an orbital Poincaré sphere proposed by Padgett and Courtial [8]. Later, this phase was experimentally demonstrated by Galvez and co-workers through interferometric measurements [9]. The geometric phase on entangled bipartite systems was extensively discussed in Refs. [10 13]. The topological structure of the geometric phase acquired by entangled quantum states under cyclic evolutions was investigated by P. Milman and R. Mosseri 14, 15], where they studied the role played by the topology of the $\mathrm{SO}(3)$ group in connection with maximally entangled states (MES). The elements of the $\mathrm{SO}(3)$ group can be represented on a parameter space with nontrivial topology, a sphere of radius $\pi$ and its diametrically opposite points identified. Two homotopy classes of closed trajectories can be identified in this topology. One is composed by closed trajectories that cross the surface of the sphere an even number of times, which we shall designate as the 0 -class ones. Closed trajectories that cross the surface of the sphere an odd number of times will be called the $\pi$-class ones. Each point of the $\mathrm{SO}(3)$ parameter space can be associated to a maximally entangled state as in ref. [16]. The relevance of this parameterization is evidenced when the geometric phase is computed for cyclic transformations performed on maximally entangled states. The topological phase acquired by a maximally entangled state is 0 for 0 -class and $\pi$ for $\pi$-class trajectories. This effect was observed almost simultaneously in two different systems, spin-orbit transformations on a laser beam [17. and two qubit manipulation by nuclear magnetic resonance (NMR) [18]. Later, the topological phases were generalized to pairs of qudits of any dimension [19] and multiple qubit systems [20], where fractional phase values were predicted. These fractional topological phases can in principle be measured in spatial qubits and qudits encoded in quantum correlated photons generated by spontaneous parametric down conversion [21, 22].

The study of vector vortex beams as non separable spin-orbit modes, benefit from several concepts of quantum entanglement theory. One remarkable example is the representation of maximally entangled states in the doubly connected manifold associated with the elements of the $\mathrm{SO}(3)$ group, which also holds for vector vortices. This representation was used in Ref.[17] to demonstrate the discrete topological phases acquired by a vector vortex beam under polarization transformations. In the present work we improved our experimental result on spin-orbit laser modes by implementing a trajectory where both polarization (spin) and orbital transformations are performed. Also, we avoided paths lying on the surface of the $\mathrm{SO}(3)$ sphere, where the representation is singular. Now the $\pi$-class trajectory implemented crosses the surface of the sphere on a single point. Our present results generalize the previous ones of Ref.[17].

\section{SPIN-ORBIT MODE STRUCTURE}

In many textbooks, the spatial mode structure produced by laser resonators are usually described by solutions of the paraxial wave equation 23, 24]. These solutions are represented by Hermite-Gaussian (HG) functions in Cartesian coordinates and by Laguerre-Gaussian (LG) functions in cylindrical coordinates. Both families are characterized by a pair of integer indexes and can be cast in a hierarchy of different orders, starting with the zero order composed solely by the fundamental Gaussian beam. In one of the seminal works describ- 
ing linear transformations between the two set of modes, Abramochkin and Volostnikov showed how the two families of modes are connected. In particular, the first order subspace is isomorphous to polarization modes, what allowed Padgett and Courtial to define an orbital Poincaré sphere, where the first order LG modes with \pm 1 helicity play the role of right and left circularly polarized light, being represented on the poles of the sphere. The HG modes with all possible orientations play the role of linear polarization states and are represented along the equator. A first order LG mode propagating along the $z$ direction is described by the following wave functions

$$
\begin{aligned}
\psi_{ \pm}(\rho, \varphi, z) & =\frac{2}{\sqrt{\pi}} \frac{\rho}{w^{2}(z)} \exp \left(-\frac{\rho^{2}}{w^{2}(z)}\right) \\
& \times \exp \left\{i\left[\frac{k \rho^{2}}{2 R(z)}+2 \arctan \left(\frac{z}{z_{R}}\right)\right]\right\} e^{ \pm i \varphi}
\end{aligned}
$$

where $(\rho, \varphi)$ are the polar coordinates on the transverse plane, $z_{R}$ is the Rayleigh length, $R(z)=\left(z_{R}^{2}+z^{2}\right) / z$ is the wave front radius, and $w(z)=\sqrt{2\left(z_{R}^{2}+z^{2}\right) /\left(k z_{R}\right)}$ is the beam diameter at position $z$. In Cartesian coordinates, the paraxial wave equation gives rise to the so called Hermite-Gaussian (HG) modes. The first order modes are

$$
\begin{aligned}
\psi_{h}(x, y, z) & =\sqrt{\frac{2}{\pi}} \frac{2 x}{w^{2}(z)} \exp \left(-\frac{x^{2}+y^{2}}{w^{2}(z)}\right) \\
& \times \exp \left\{i\left[\frac{k\left(x^{2}+y^{2}\right)}{2 R(z)}+2 \arctan \left(\frac{z}{z_{R}}\right)\right]\right\} \\
\psi_{v}(x, y, z) & =\sqrt{\frac{2}{\pi}} \frac{2 y}{w^{2}(z)} \exp \left(-\frac{x^{2}+y^{2}}{w^{2}(z)}\right) \\
& \times \exp \left\{i\left[\frac{k\left(x^{2}+y^{2}\right)}{2 R(z)}+2 \arctan \left(\frac{z}{z_{R}}\right)\right]\right\} .
\end{aligned}
$$

One easily verifies that the first order LG and HG modes are connected by the following simple relation

$$
\psi_{ \pm}=\frac{\psi_{h} \pm i \psi_{v}}{\sqrt{2}} .
$$

HG modes can also be defined on rotated coordinates systems. For example, the HG modes oriented at $\pm 45^{\circ}$ are given by

$$
\psi_{ \pm 45^{\circ}}=\frac{\psi_{h} \pm \psi_{v}}{\sqrt{2}}
$$

Therefore, if we combine the structure of first order paraxial modes with polarization, we can build spin-orbit modes and establish the analogy with two-qubit systems in quantum mechanics. For example, cylindrically polarized beams have the same mathematical structure of the so called Bell states, defined in connection with Bell inequalities. In terms of $\mathrm{HG}$ and linear polarization modes

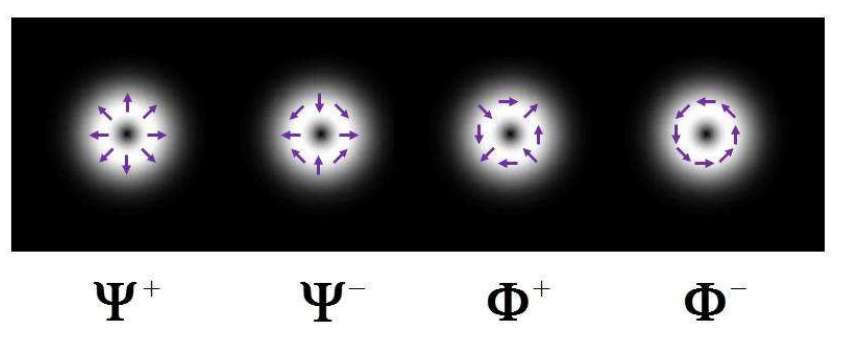

FIG. 1: Linear polarization patterns of cylindrically polarized beams.

they are given by

$$
\begin{aligned}
& \boldsymbol{\Psi}^{ \pm}=\frac{\psi_{h} \hat{\mathbf{e}}_{H} \pm \psi_{v} \hat{\mathbf{e}}_{V}}{\sqrt{2}}, \\
& \boldsymbol{\Phi}^{ \pm}=\frac{\psi_{h} \hat{\mathbf{e}}_{V} \pm \psi_{v} \hat{\mathbf{e}}_{H}}{\sqrt{2}} .
\end{aligned}
$$

where $\hat{\mathbf{e}}_{H}$ and $\hat{\mathbf{e}}_{V}$ are the linear polarization unit vectors along the horizontal and vertical directions, respectively. These modes correspond to the linear polarization patterns depicted in Fig.(1). They have been employed in a number of optical experiments both in applied and fundamental subjects [25]. Their connection with Bell inequalities both in classical and quantum optics has been discussed in Refs. 26 31] and their use on alignment free quantum cryptography was investigated in Refs. 32 34.

For our experimental context, it will be particularly useful to employ the LG basis for the spatial modes, since their characteristic phase singularity will work as a spatial reference for our interferometric measurements. Therefore, it will be convenient to write a general first order spin-orbit mode as:

$$
\begin{aligned}
\boldsymbol{\Psi} & =\lambda_{1} \psi_{+} \hat{\mathbf{e}}_{H}+\lambda_{2} \psi_{-} \hat{\mathbf{e}}_{H}+\lambda_{3} \psi_{+} \hat{\mathbf{e}}_{V}+\lambda_{4} \psi_{-} \hat{\mathbf{e}}_{V} \\
& \equiv\left[\lambda_{1}, \lambda_{2}, \lambda_{3}, \lambda_{4}\right]^{T} .
\end{aligned}
$$

The separability of a spin-orbit mode can be quantified by the analogous definition of concurrence. For the spinorbit mode described by Eq.(17), the concurrence is

$$
C=2\left|\lambda_{1} \lambda_{4}-\lambda_{2} \lambda_{3}\right|
$$

\section{THE EXPERIMENT}

In order to compute the whole sequence of spin-orbit mode transformations, it is essential first to define the operators that describe the spatial and polarization mode conversions. We will be dealing with wave plates for polarization and astigmatic mode converters for the transverse mode transformations, that is, elements acting on each degree of freedom separately. We will express rotation angles in degrees and phase retardations in radians 
for immediate identification of their physical meaning. When their fast axis is oriented along the horizontal direction, wave plates introduce a retardation phase $\phi$ between $H$ and $V$ polarizations. For example, a quarter wave plate (QWP) corresponds to $\phi=\pi / 2$ and a half wave plate (HWP) to $\phi=\pi$. When the fast axis is rotated by the angle $\theta$, its action in the $\left\{\hat{\mathbf{e}}_{H}, \hat{\mathbf{e}}_{V}\right\}$ basis is described by the following $\mathrm{SU}(2)$ matrix

$W(\theta, \phi)=\left[\begin{array}{cc}\cos \frac{\phi}{2}+i \sin \frac{\phi}{2} \cos 2 \theta & i \sin \frac{\phi}{2} \sin 2 \theta \\ i \sin \frac{\phi}{2} \sin 2 \theta & \cos \frac{\phi}{2}-i \sin \frac{\phi}{2} \cos 2 \theta\end{array}\right]$.

Spatial mode converters can be made with cylindrical lenses [35 38] for variable retardation $\phi$, or the DP for $\phi=\pi$. They act on spatial modes $\psi_{h}$ and $\psi_{v}$ in the same way the wave plates act on horizontal and vertical polarization. However, as stated above, we will use the $\left\{\psi_{+}, \psi_{-}\right\}$basis for the spatial modes. In this basis a spatial mode converter introducing a retardation $\phi$, and rotated by the angle $\theta$ is described by the $\mathrm{SU}(2)$ matrix

$$
C(\theta, \phi)=\left[\begin{array}{cc}
\cos \frac{\phi}{2} & i \sin \frac{\phi}{2} e^{-2 i \theta} \\
i \sin \frac{\phi}{2} e^{2 i \theta} & \cos \frac{\phi}{2}
\end{array}\right] .
$$

The mode preparation is sketched in Fig,2, A linearly polarized $\mathrm{TEM}_{00}$ mode from a frequency doubled Nd:YAG laser $(\lambda=532 \mathrm{~nm})$ is transmitted through a radial polarization converter (S-Wave Plate, Model ALTECHNA RPC-515-04) that prepares the initial $\boldsymbol{\Psi}^{+}$ mode. Before sending it through the transformation sequence, the quality of the radially polarized beam is checked with a half wave plate and a polarizer beam splitter (not shown in the figure). When the beam is well prepared, we observe a rotating HG mode transmitted through the polarizing beam splitter, as we rotate the wave plate. Then, the test elements are removed from the setup and the beam is sent through the transformation sequence. The $\boldsymbol{\Psi}^{+}$mode prepared by the S-plate is sent through a quarter wave plate (QWP-1) oriented at $0^{\circ}$ and a half wave plate (HWP-1) oriented at $22.5^{\circ}$. Then, a spatial filter is used to improve the beam shape. This sequence prepares the initial nonseparable mode

$$
\begin{aligned}
\boldsymbol{\Psi}_{0} & =\frac{\psi_{+} \hat{\mathbf{e}}_{H}+\psi_{-} \hat{\mathbf{e}}_{V}}{\sqrt{2}} \\
& \equiv \frac{1}{\sqrt{2}}[1,0,0,1]^{T},
\end{aligned}
$$

for which $C=1$. Separable modes were produced by filtering one of the polarization components with a polarizing beam splitter (PBS). Therefore, the initial mode can be described by the general formula

$$
\begin{aligned}
\boldsymbol{\Psi}_{i} & =\sqrt{\epsilon} \psi_{+} \hat{\mathbf{e}}_{H}+\sqrt{1-\epsilon} \psi_{-} \hat{\mathbf{e}}_{V} \\
& \equiv[\sqrt{\epsilon}, 0,0, \sqrt{1-\epsilon}]^{T},
\end{aligned}
$$

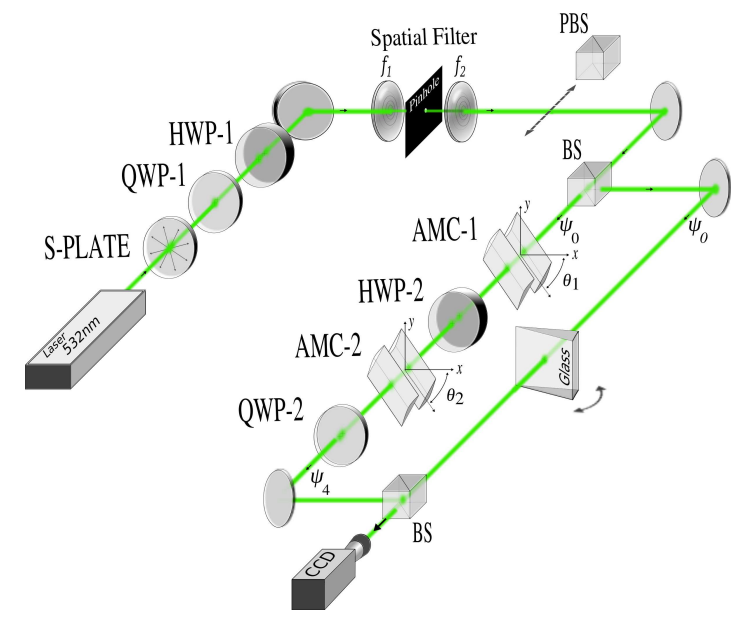

FIG. 2: Experimental setup.

where $\epsilon=0$ when the horizontal polarization is blocked, 1 when the vertical polarization is blocked and $1 / 2$ when both polarization are present.

Let us describe the transformation sequence performed on mode $\boldsymbol{\Psi}_{0}$, that is when $\epsilon=1 / 2$. The probe beam is sent through a Mach-Zehnder (MZ) interferometer used for the topological phase measurement. In one arm of the interferometer, the initial mode is used as a reference beam. In the other arm, a sequence of spatial and polarization mode transformations is implemented. First, the initial spin-orbit mode $\Psi_{0}$ is transmitted through a $\pi / 2$ astigmatic mode converter (AMC-1) oriented at $22.5^{\circ}$ that produces mode

$$
\begin{aligned}
\boldsymbol{\Psi}_{1} & =\left[\mathbb{1} \otimes C\left(22.5^{\circ}, \pi / 2\right)\right] \mathbf{\Psi}_{0} \\
& =\frac{1}{2}\left[1, e^{\frac{3 i \pi}{4}}, e^{\frac{i \pi}{4}}, 1\right]^{T} .
\end{aligned}
$$

A half wave plates (HWP-2) with a variable orientation $\theta$, produces mode

$$
\begin{aligned}
\mathbf{\Psi}_{2} & =[W(\theta, \pi) \otimes \mathbb{1}] \mathbf{\Psi}_{1} \\
& =\frac{1}{2}\left[\begin{array}{ccc}
i \cos 2 \theta & - & \sin 2 \theta e^{-\frac{i \pi}{4}} \\
-\cos 2 \theta e^{\frac{i \pi}{4}} & + & i \sin 2 \theta \\
i \sin 2 \theta & + & \cos 2 \theta e^{-\frac{i \pi}{4}} \\
-\sin 2 \theta e^{\frac{i \pi}{4}} & - & i \cos 2 \theta
\end{array}\right] .
\end{aligned}
$$

The orientation $\theta$ is varied between $-45^{0}$ and $+45^{0}$ to provide a 0 or $\pi$-class trajectory, respectively. A second $\pi / 2$ mode converter (AMC-2) also oriented at $22.5^{\circ}$ generates

$$
\begin{aligned}
\boldsymbol{\Psi}_{3} & =\left[\mathbb{1} \otimes C\left(22.5^{\circ}, \pi / 2\right)\right] \boldsymbol{\Psi}_{2} \\
& =\frac{1}{\sqrt{2}}\left[\begin{array}{cc}
-\sin 2 \theta & e^{-\frac{i \pi}{4}} \\
-\cos 2 \theta & e^{\frac{i \pi}{4}} \\
\cos 2 \theta & e^{-\frac{i \pi}{4}} \\
-\sin 2 \theta & e^{\frac{i \pi}{4}}
\end{array}\right],
\end{aligned}
$$


and a quarter wave plate (QWP-2) oriented at $0^{\circ}$ makes the final transformation to

$$
\begin{aligned}
\boldsymbol{\Psi}_{4} & =\left[W\left(0^{\circ}, \pi / 2\right) \otimes \mathbb{1}\right] \boldsymbol{\Psi}_{3} \\
& =\frac{1}{\sqrt{2}}\left[\begin{array}{ll}
- & \sin 2 \theta \\
-i & \cos 2 \theta \\
-i & \cos 2 \theta \\
- & \sin 2 \theta
\end{array}\right] .
\end{aligned}
$$

From (16) we immediately see that the complete transformation sequence is cyclic when $\theta= \pm 45^{0}$, giving $\boldsymbol{\Psi}_{4}= \pm \boldsymbol{\Psi}_{0}$.

In order to draw the trajectories in the $\mathrm{SO}(3)$ sphere, we will use the parameterization detailed in ref. 39]. An arbitrary maximally non separable mode can be written as

$$
\begin{aligned}
\boldsymbol{\Psi}_{N S} & =\frac{1}{\sqrt{2}}\left(\lambda \psi_{+} \hat{\mathbf{e}}_{H}+\eta \psi_{-} \hat{\mathbf{e}}_{H}-\eta^{*} \psi_{+} \hat{\mathbf{e}}_{V}+\lambda^{*} \psi_{-} \hat{\mathbf{e}}_{V}\right) \\
& \equiv \frac{1}{\sqrt{2}}\left[\lambda, \eta,-\eta^{*}, \lambda^{*}\right]^{T} .
\end{aligned}
$$

The point representing mode (17) in the $\mathrm{SO}(3)$ sphere is localized by the vector $\mathbf{v}=a \hat{\mathbf{u}}$, where $a \in[0, \pi]$ is the distance from the origin of the sphere and $\hat{\mathbf{u}}$ is the unit vector oriented from the origin to the point representing the corresponding mode. Following ref. 39], the mode coefficients and the point coordinates are related by

$$
\begin{aligned}
\lambda & =\cos \frac{a}{2}-i u_{z} \sin \frac{a}{2}, \\
\eta & =-\left(u_{y}+i u_{x}\right) \sin \frac{a}{2} .
\end{aligned}
$$

Mode $\boldsymbol{\Psi}_{0}$ is represented by the origin of the $\mathrm{SO}(3)$ sphere. Therefore, for $\theta= \pm 45^{0}$, the mode transformations are represented by the following points $\left(a, u_{x}, u_{y}, u_{z}\right)$ in the sphere

$$
\begin{aligned}
\boldsymbol{\Psi}_{0} & \equiv \boldsymbol{\Psi}_{4} \equiv(0,0,0,1), \\
\boldsymbol{\Psi}_{1} & \equiv\left(\frac{\pi}{2},-\frac{1}{\sqrt{2}}, \frac{1}{\sqrt{2}}, 0\right), \\
\boldsymbol{\Psi}_{2} & \equiv\left(\frac{2 \pi}{3}, \sqrt{\frac{2}{3}}, 0, \frac{1}{\sqrt{3}}\right), \\
\boldsymbol{\Psi}_{3} & \equiv\left(\frac{\pi}{2}, 0,0,1\right) .
\end{aligned}
$$

These points are shown in Fig.(3) together with the alternative paths connecting them. When HWP-2 is set to $\theta=-45^{0}$, the 0 -class trajectory indicated in blue (online) is followed from $\boldsymbol{\Psi}_{1}$ to $\boldsymbol{\Psi}_{2}$. When HWP-2 is set to $\theta=45^{0}$, the $\pi$-class trajectory indicated in white is followed, where the surface of the $\mathrm{SO}(3)$ sphere is crossed at point $P$ and the trajectory continues from the opposite point $P^{\prime}$. The $\pi$ phase shift becomes clear when we compare Eqs. (11) and (16) for each value of $\theta$. In order to evidence this phase shift experimentally, we need to interfere the transformed beam with a reference.

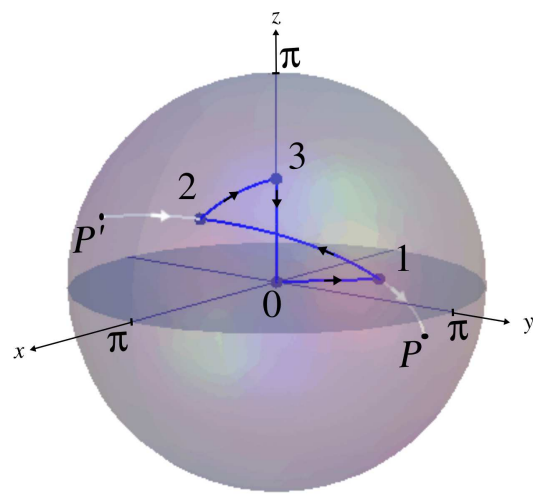

FIG. 3: Diagram representing the transformations performed in the $S O(3)$ sphere. The 0 -class path is indicated in blue (online) and the $\pi$-class path between points 1 and 2 is indicated in white.

The two arms of the interferometer are slightly misaligned to provide spatial interference. We can calculate the expected interference pattern assuming the general initial spin-orbit mode given by Eq.(12),

$$
\begin{aligned}
I(\mathbf{r}) & =\left|\Psi_{4}(\mathbf{r})+e^{i \mathbf{q} \cdot \mathbf{r}} \Psi_{0}(\mathbf{r})\right|^{2}, \\
& =F(\rho, z)[1-2 \sqrt{\epsilon(1-\epsilon)} \sin 2 \theta \cos (\mathbf{q} \cdot \mathbf{r}) \\
& -\epsilon \cos 2 \theta \sin (\mathbf{q} \cdot \mathbf{r}+2 \varphi) \\
& -(1-\epsilon) \cos 2 \theta \sin (\mathbf{q} \cdot \mathbf{r}-2 \varphi)],
\end{aligned}
$$

where $\mathbf{q}$ is the transverse wavector difference between the misaligned beams and

$$
F(\rho, z)=\frac{4 \rho^{2}}{\pi w^{4}(z)} \exp \left(-\frac{2 \rho^{2}}{w^{2}(z)}\right)
$$

is the radially symmetric intensity distribution of the Laguerre-Gaussian modes. Eq.(20) allows us to predict the effects of the parameters involved in the experimental setup. When HWP-2 is oriented at $\theta= \pm 45^{0}$ (the settings leading to closed trajectories in the $\mathrm{SO}(3)$ sphere), the last two terms in the square brackets vanish and the interference pattern is determined solely by the second term with the fringes visibility given by

$$
\mathcal{V}\left(\theta= \pm 45^{0}\right)=|2 \sqrt{\epsilon(1-\epsilon)}| .
$$

Therefore, maximal visibility is expected for $\epsilon=1 / 2$ and no interference fringes are expected for separable modes $(\epsilon=0,1)$. This behavior was experimentally confirmed, as can be seen from the interference patterns shown in Fig.(4). The upper left and right corners of this figure correspond to the interference patterns obtained with the non separable mode for the 0 and $\pi$ classes of closed trajectories. The $\pi$ phase shift is clear from the location of the phase singularity present in the LG modes, which is used as a spatial reference. For $\theta=-45^{0}$, the singularity is located on a bright fringe while for $\theta=45^{0}$ 


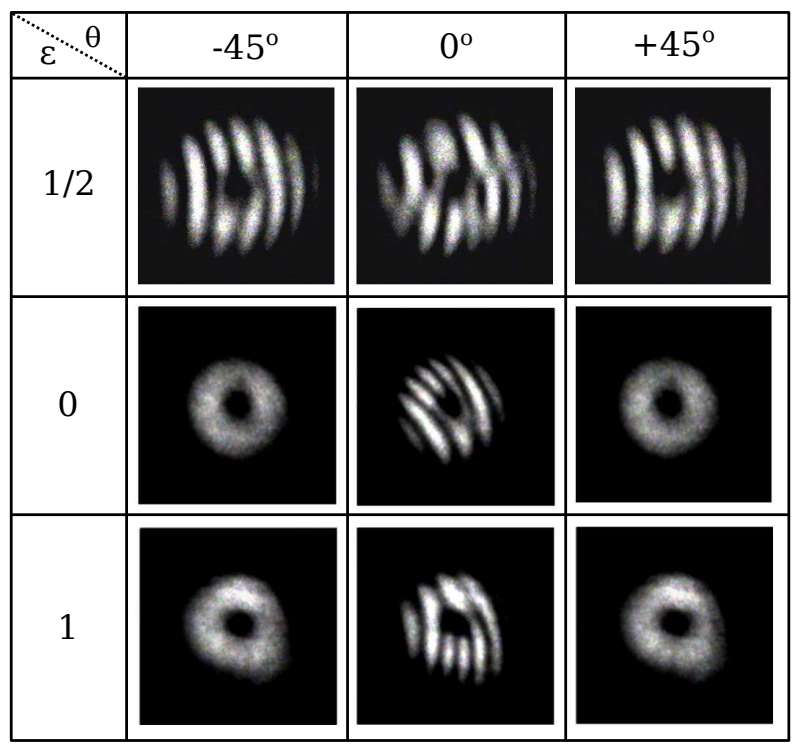

FIG. 4: Images of the interference pattern observed at the output of the Mach-Zehnder interferometer. The corresponding values of $\epsilon$ and $\theta$ are indicated.

it falls on a dark fringe. The interferometer was stable over several minutes, more than enough for image acquisition and rotation of the wave plate from $-45^{0}$ to $45^{0}$ in steps of $22.5^{0}$, so that no active stabilization was required. The separable modes were obtained by selecting each polarization component with a polarizing beam splitter (PBS). As expected, no interference fringes are seen when $\theta= \pm 45^{\circ}$, what can be verified in the left and right corners of the middle and bottom rows of Fig.(4).

We could also simulate the whole set of interference patterns in a density plot of the theoretical expression (20). The corresponding results are shown in Fig.(5). The only fitting parameters are the components of the transverse wave vector $\mathbf{q}$, which were set to provide orientation and frequency of the interference fringes similar to those of the experimental patterns. The theoretical images exhibit a fairly good agreement with the experimental patterns in regards to the fringes visibility and their geometrical structure. The experimental patterns are slightly curved due to a small wave front mismatch in the Mach-Zehnder interferometer, not simulated in the theoretical images. The $\pi$ phase shift of the nonseparable mode is also evident from the displacement of the interference fringes with respect to the location of the phase singularity.

\section{CONCLUSION}

In conclusion, we investigated the topological phase acquired by vector vortex beams under combined local unitary operations on the polarization and transverse

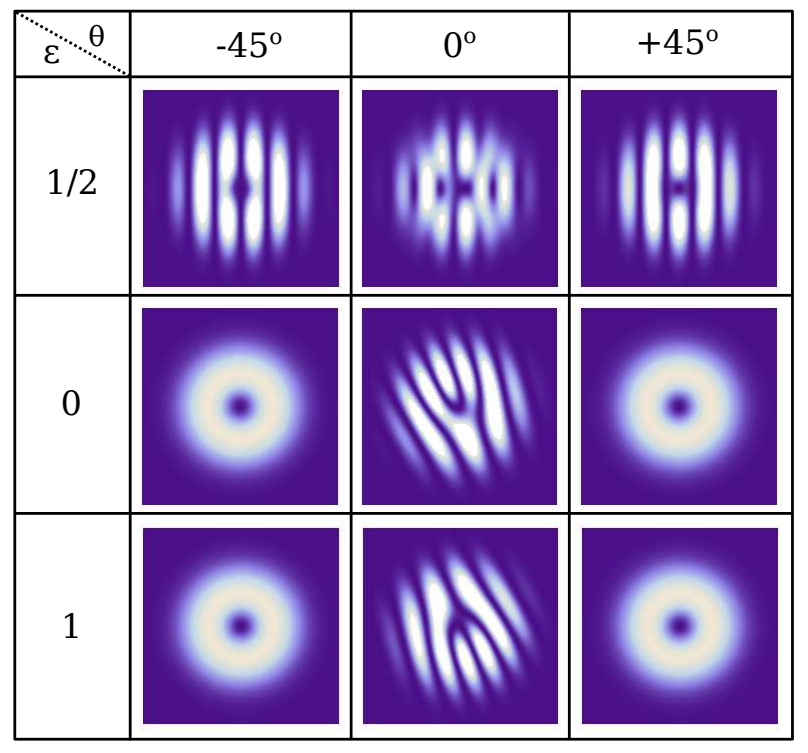

FIG. 5: Density plot of Eq. (20), giving the theoretical images of the interference patterns at the output of the Mach-Zehnder interferometer. The images are in one to one correspondence with those in Fig 4

mode degrees of freedom. The representation of maximally nonseparable spin-orbit modes in the $\mathrm{SO}(3)$ manifold gives a crucial role to the doubly connected topology of the group in the interpretation of the possible phase values acquired under cyclic evolutions. Our measurements involve operations on both degrees of freedom in a Mach-Zehnder interferometer, and the role of the mode separability could be captured through the visibility of the interference patterns.

Funding was provided by Coordenação de Aperfeiçoamento de Pessoal de Nível Superior (CAPES), Fundação de Amparo à Pesquisa do Estado do Rio de Janeiro (FAPERJ-BR), and Instituto Nacional de Ciência e Tecnologia de Informação Quântica (INCT-IQ$\mathrm{CNPq})$.

[1] S. Pancharatnam, Proc. Indian Acad. Sci., Sect. A 44, 247 (1956).

[2] Collected Works of S. Pancharatnam, Oxford Univ. Press, London (1975).

[3] Y. Aharonov and D. Bohm, Phys. Rev. 115, 485 (1959).

[4] M. V. Berry, Proc. R. Soc. London A 392, 45 (1984).

[5] J. A. Jones et al. , Nature (London) 403, 869 (2000).

[6] L.-M. Duan, J. I. Cirac, and P. Zoller, Science 292, 1695 (2001).

[7] S.J. van Enk, Opt. Comm. 102, 59 (1993).

[8] M. J. Padgett and J. Courtial, Opt. Lett. 24, 430 (1999).

[9] E. J. Galvez et al, Phys. Rev. Lett. 90, 203901 (2003).

[10] E. Sjöqvist, Phys. Rev. A 62, 022109 (2000).

[11] B. Hessmo, E. Sjöqvist, Phys. Rev. A 62, 062301 (2000). 
[12] D. M. Tong, E. Sjöqvist, L. C. Kwek, C. H. Oh, M. Ericsson, Phys. Rev. A 68, 022106 (2003).

[13] E. Sjöqvist, Physics Letters A 374, 1431 (2010).

[14] P. Milman, and R. Mosseri, Phys. Rev. Lett. 90, 230403 (2003).

[15] P. Milman, Phys. Rev. A 73, 062118 (2006).

[16] R. Mosseri, and R. Dandoloff, J. Phys. A 34, 10243 (2003).

[17] C. E. R. Souza, J. A. O. Huguenin, P. Milman, A. Z. Khoury, Phys. Rev. Lett. 99, 160401 (2007).

[18] J. Du, J. Zhu, M. Shi, X. Peng, and D. Suter, Phys. Rev. A 76, 042121 (2007).

[19] L. E. Oxman and A. Z. Khoury, Phys. Rev. Lett. 106, 240503 (2011).

[20] M. Johansson, M. Ericsson, K. Singh, E. Sjöqvist, and M. S. Williamson, Phys. Rev. A 85, 032112 (2012).

[21] A. Z. Khoury, L. E. Oxman, B. Marques, A. Matoso, and S. Pádua, Phys. Rev. A 87, 042113 (2013).

[22] M. Johansson, A. Z. Khoury, K. Singh, E. Sjöqvist, Phys. Rev. A 87, 042112 (2013).

[23] A. E. Siegman, Lasers, University Science Books (1986).

[24] A. Yariv, Quantum Electronics, John Wiley \& Sons, third ed. (1988).

[25] C. Gabriel, A. Aiello, W. Zhong, T. G. Euser, N. Y. Joly, P. Banzer, M. Fortsch, D. Elser, U. L. Andersen, C. Marquardt, P. S. J. Russell, G. Leuchs, Phys. Rev. Lett. 106, 060502 (2011).

[26] R. J. C. Spreeuw, Found. of Phys. 28361 (1998).
[27] R. J. C. Spreeuw, Phys. Rev. A 63, 062302 (2001).

[28] C. V. S. Borges, M. Hor-Meyll, J. A. O. Huguenin, and A. Z. Khoury, Phys. Rev. A 82, 033833 (2010).

[29] L. Chen and W. She, J. Opt. Soc. Am. B, 27, A7 (2010).

[30] E. Karimi, J. Leach, S. Slussarenko, B. Piccirillo, L. Marrucci, L. Chen, W. She, S. Franke-Arnold, M. J. Padgett, E. Santamato, Phys. Rev. A 82, 022115 (2010).

[31] K. H. Kagalwala, G. Di Giuseppe, A. F. Abouraddy, B. E. A. Saleh, Nature Photonics 7, 72 (2013).

[32] L. Aolita and S. P. Walborn, Phys. Rev. Lett. 98, 100501 (2007).

[33] C. E. R. Souza, C. V. S. Borges, A. Z. Khoury, J. A. O. Huguenin, L. Aolita, and S. P. Walborn, Phys. Rev. A 77, 032345 (2008).

[34] V. D'Ambrosio, E. Nagali, S. P. Walborn, L. Aolita, S. Slussarenko, L. Marrucci, F. Sciarrino, Nature Communications 3, 961 (2012).

[35] E. Abramochkin and V. Volostnikov, Opt. Commun. 83, 123(1991)

[36] L. Allen, M. W. Beijersbergen, R. J. C. Spreeuw, and J. P. Woerdman, Phys. Rev. A 45, 8185 (1992).

[37] M. W. Beijersbergen, L. Allen, H. E. L. O. var der Veen, and J. P. Woerdman, Opt. Commun. 96, 123 (1992).

[38] C. E. R. Souza and A. Z. Khoury, Opt. Express 18, 9207 (2010).

[39] W. LiMing, Z. L. Tang, and C. J. Liao, Phys. Rev. A 69, 064301 (2004). 